\title{
The modulatory influence of different phases of menstrual cycle on the parasympathetic tone in sedentary females-A cross-sectional study
}

\author{
Nirmala S. Anand ${ }^{1, *}$, Shivaprasad Goudar ${ }^{2}$ \\ ${ }^{1}$ Assistant Professor, ${ }^{2}$ Professor, Dept. of Physiology, Jawaharlal Nehru Medical College, Belgaum, Karnataka, India \\ * Corresponding author: \\ Email:drnirm79@gmail.com
}

Received: $28^{\text {th }}$ September, 2017

Accepted: $16^{\text {th }}$ November, 2017

\begin{abstract}
Introduction: The cyclical fluctuating gonadal hormones in a female affect the functioning of the autonomic nervous system \& a decline in parasympathetic neural activity is a forerunner to multiple cardiac conditions like MI, angina etc hence its detection at its inception is primary to institute remedial measures.

Aim: The aim of the present study was to examine whether the cardiac parasympathetic status of a healthy eumenorrhic female is altered during different phases of menstrual cycle.

Materials and Methods- Thirty eumenorrhic females were recruited during the follicular and luteal phases of menstrual cycle \& subjected to battery of parasympathetic autonomic tests

Statistical Analysis: Data was analyzed statistically using student's paired t-test.

Results: The results from 30:15 ratio in follicular phase was (1.19 \pm 0.05$)$ while in luteal (1.08 \pm 0.05$)$, E:I ratio in follicular (1.58 $\pm 0.15)$ while in luteal phase was $(1.29 \pm 0.25)$, Valsalva ratio in follicular $(1.89 \pm 0.32)$ while in luteal phase was $(1.73 \pm 0.34)$ while the values of DBD and Tachycardia ratio were insignificant statistically

Conclusion: We hence conclude that there is an obvious interaction between the gonadal hormones and autonomic nervous system which is reflected by a reduced vagal outflow during luteal phase.
\end{abstract}

Keywords: Autonomic, Deep Breathing difference, Menstrual cycle, Parasympathetic, Vagal.

\section{Introduction}

The menstrual cycle is an endogenous biological rhythm where there are cyclical changes in the reproductive organs as well as many other organ systems as a result of fluctuations in the levels of gonadal hormonesnamely estrogen and progesterone which are secreted as a result of co-ordinated action of hypothalamo-pituitary-gonadal axis. ${ }^{1-3}$ Maintenance of normal homeostasis before or after encountering a stressful situation involves a pivotal role of the autonomic nervous system which acts through its two effector arms i.e. sympathetic and parasympathetic forming a controlled loop with the brain and spinal cord which regularly controls the set point of the system. ${ }^{1,2}$ The gonadal hormones have discrete interactions with the autonomic centers in the brain, medulla as well as HPA axis. There are reports cited in literature of the interaction of the gonadal hormones with the autonomic nervous system. There is no clear cut consensus regarding the interaction of the endogenous gonadal hormones with the ANS, but the presence of estrogen receptors in the medulla namely the tractus solitarius which modulates cardiorespiratory activities, heart suggest some kind of interaction between both of them. ${ }^{4}$ Parasympathetic activity is primary in regulating the SA node which is the pacemaker of the heart because of its rapidity of action and rapid decay compared to its counterpart the sympathetic nervous activity which has a long latency and takes considerable amount of time for termination of its action. ${ }^{1,2}$ A study reporting no significant change in the ANS activity through the menstrual cycle using the heart rate measurement using ECG \& phonocardiography. ${ }^{5}$ Contradictory results have been reported by a study wherein they have observed an increase in the parasympathetic activity during the secretory phase while no change was associated with respect to sympathetic activity during any phase of menstrual cycle. ${ }^{6}$ Several noninvasive tests to incite the ANS have been devised where recording response changes in the cardiovascular system in the form of heart rate change or change in blood pressure to standardized stimuli has been introduced..$^{7-11}$ Autonomic imbalance primarily in the formof vagal dysfunction precedes the onset of chronic diseases involving the cardiovascular, neurological, reproductive system. ${ }^{7}$ Hence testing for the autonomic status of an individual is worthwhile since most of these disorders can lend themselves to preventive or early curative strategies. Hence the study was designed to focus on the interaction of the ANS with the gonadal hormones and to identify the lacunae which exist in literature.

\section{Objective}

The objective of this study was to assess whether different phases of menstrual cycle actually affect the functioning of the cardiacparasympathetic outflow 


\section{Materials and Methods}

Study Design: Cross-sectional

Duration: December 2011 till February 2012

Source of Data: In the present study the data was collected from the students of first year M.B.B.S for the academic year 2011.

Sample Size: Thirty eumenorrhic girls aged $17 \quad-20$ years of age studying their first year MBBS course were selected

\section{Sample Size Estimation}

Expected Reduction $-($ MEAN $)=\mathrm{d}=20$

$\mathrm{SD}=40=\sigma$

$\alpha=0.05 \quad$ One sided $\quad \mathrm{Z} \alpha \dot{\alpha}=1.65$

$\beta=0.2 \quad$ Power $80 \% \quad Z \beta=0.84$

$\mathrm{n}=[(\mathrm{Z} \dot{\alpha}+\mathrm{Z} \beta) \sigma / \mathrm{d}]^{2}=24.8=25=30$

Based on sample size calculation 30 MBBS phase 1 females aged 17-20 years who are eligible were enrolled at the time of data collection.

Inclusion Criteria: Thirty eumenorrhic girls aged 17 20 years of age studying their MBBS course with regular menstrual cycle duration of 28days at least 2 months prior to the study, having no medical or gynecological problems, no well-defined premenstrual tension were enrolled.

Exclusion criteria: Subjects who had irregular menstrual cycles, dysmenorrhea, menorrhagia, had any endocrine disorder or consuming over the counter drugs like cough suppressants, antidepressant, oral contraceptive pill, hormonal replacement therapy, drugs that alter the cardiovascular functions were excluded from the study. Females engaging in active exercise i.e.-3 times/week are excluded from the study since the work extends to sedentary females.

Voluntary Informed written consent was obtained from all participants, and the experiment protocol was approved by Ethics committee of the college. A detailed menstrual history which included the no of days, regularity and total duration of cycle was collected prior to enrollment of every participant.

The entire purpose along with a detailed description of the study protocol was given to all the subjects prior to beginning of the test\& examination carried out at same time of the day.

Considering the first day of bleeding as Day 1, the phases marked out were and confirmed by basal body temperature monitoring:

1. Follicular $\left(10^{\text {th }}\right.$ day $)$

2. Luteal $\left(20^{\text {th }}\right.$ day).

Parasympathetic Measures: $:^{5,6}$

\begin{tabular}{|l|}
\hline Parasympathetic tests \\
\hline 1) 30:15 ratio(Orthostatic Tolerance Test) \\
\hline 2) E:I ratio \\
\hline 3) Deep Breathing Difference (DBD) \\
\hline
\end{tabular}

4)) Valsalva index

5) Tachycardia ratio

1. 30:15 Ratio (Orthostatic Tolerance Test): is considered as a measure of cardiac vagal function. With change in the posture from supine to standing causes venous pooling leading to hydrostatic stress which activates the baroreflex leading to increased heart rates within 10-20 seconds of assuming upright posture and returns to baseline after 25-30 seconds.

Procedure: Resting ECG was recorded and baseline heart rate measured.

The subject was asked to stand with support to avoid the heart muscle reflex and a continuous ECG is recorded for 1-3 minutes.

\section{0:15 Ratio}

Longest RR interval 30 beats after standing Shortest RR interval 15 beats after standing.

Normal value->1.04

2. Sinus Arrhythmia: The variation in heart rate with deep breathing i.e. -increase in heart rate during inspiration while decrease during expiration is known as Sinus arrhythmia. In supine position the subject was asked to breathe slowly and deeply at the rate of 6 to 8 breaths/minute, inspiration and expiration lasting for 5 seconds each which was set using a metronome\& ECG was continuously recorded. The ratio of the $\mathrm{R}-\mathrm{R}$ interval during expiration versus inspiration is called the $\mathrm{E}$ : I Index.

a) E: I Ratio

Maximum R-R interval in $\mathrm{mm}$ (during expiration) Minimum R-R interval in $\mathrm{mm}$ (during inspiration)

Normal Value: E:I ratio->1.21

b) Deep Breathing Difference $=$ mean of the difference between maximum \& minimum heart rates for six measured cycles in bpm.

Normal Value: Deep Breathing Difference->18 bpm

3. Valsalva Index: For this test the subject was asked to sit and blow forcefully into mercury sphygmomanometer rubber tubing. The subject raised the mercury column to $40 \mathrm{~mm} \mathrm{Hg}$ and maintained it for at least 30 seconds during which continuous ECG tracing was recorded. The valsalva index was calculated as follows.

a) Valsalva index

Maximum R-R interval in mm(during release)

Minimum R-R interval in mm (during strain)

Normal Value-Valsalva ratio: $>1.45$

b) Tachycardia Ratio

Shortest R-R interval during maneuver

Longest R-R interval before maneuver

\section{Statistical analysis}

The data quantified as mean \pm SD and analyzed statistically using Student's paired t-test $\mathrm{p}<0.05$ was 
considered to be statistically significant Statistical software SPSS 18 version was used for the analysis of the data.

\section{Results}

The current study used the parasympathetic subtests from the Ewing \& Clarke Battery of tests. To assess the status of the parasympathetic tone we used the 30:15 ratio, E:I ratio, Deep Breathing Difference, Valsalva ratio\& Tachycardia ratio.

Table 1: Shows the distribution of demographic variables

\begin{tabular}{|l|c|}
\hline AGE(yrs.) & $18.3 \pm 0.66(17-20)$ \\
\hline WEIGHT(kg) & $56.7 \pm 7.55(47-65)$ \\
\hline HEIGHT $\left(\mathrm{m}^{2}\right)$ & $1.56 \pm 0.04(1.51-1.68)$ \\
\hline
\end{tabular}

Table 1 shows the demographic profile of the participants. It is evident that most of the participants are of the mean age group of 18 years and average weight of $56 \mathrm{kgs}$.

Table 2: Comparison of 30:15 ratio during different phases of menstrual cycle

\begin{tabular}{|l|c|l|c|}
\hline & Follicular & Luteal & P value \\
\hline 30;15 Ratio & $1.19 \pm 0.05$ & $1.08 \pm 0.05$ & $0.001^{*}$ \\
\hline
\end{tabular}

Table 2 shows tests used to compute parasympathetic measures of autonomic function test i.e. 30:15 ratio. The table 2 shows the comparison of 30:15 during follicular and luteal phase. The values of 30:15ratio were higher in follicular phase showing parasympathetic predominance in it. $\left(\mathrm{p}=0.001^{*}\right)$

Table 3: Comparison of Sinus Arrhythmia using E:I ratio \& Deep Breathing Difference during difference phases of menstrual cycle

\begin{tabular}{|l|c|c|c|}
\hline & Follicular & Luteal & P value \\
\hline E:I Ratio & $1.58 \pm 0.15$ & $1.29 \pm 0.25$ & $0.004^{*}$ \\
\hline DBD & $31.8 \pm 6.44$ & $30.2 \pm 8.77$ & $1.70^{\mathrm{NS}}$ \\
\hline
\end{tabular}

*-statistically significant, NS-not significant

Table 3 Comparison of E:I ratio and Deep Breathing difference during follicular and luteal phases of menstrual cycle. The valueof E:I ratio was significantly higher during the follicular phase while the results of DBD show that there is no statistical difference in the values between the two phases.

Table 4: Comparison of Valsalva ratio \& Tachycardia ratio during difference phases of menstrual cycle

\begin{tabular}{|l|c|c|c|}
\hline & Follicular & Luteal & P value \\
\hline $\begin{array}{l}\text { Valsalva } \\
\text { ratio }\end{array}$ & $1.89 \pm 0.32$ & $1.73 \pm 0.34$ & $0.001^{*}$ \\
\hline $\begin{array}{l}\text { Tachycardia } \\
\text { ratio }\end{array}$ & $0.58 \pm 0.08$ & $0.56 \pm 0.07$ & $0.23^{\mathrm{NS}}$ \\
\hline
\end{tabular}

*-statistically significant, NS-not significant

Table 4 shows results of Valsalva ratio and Tachycardia ratio during follicular and luteal phases of menstrual cycle. From the results of table 4 it is evident that Valsalva ratio values were statistically higher during the follicular phase while the values of Tachycardia ratio were not significant. Hence from the above we can compute that the follicular phase shows greater vagal outflow.

Graph 1: Shows the values of various parasympathetic subtests like 30:15 ratio, E: I ratio \& Valsalva ratio

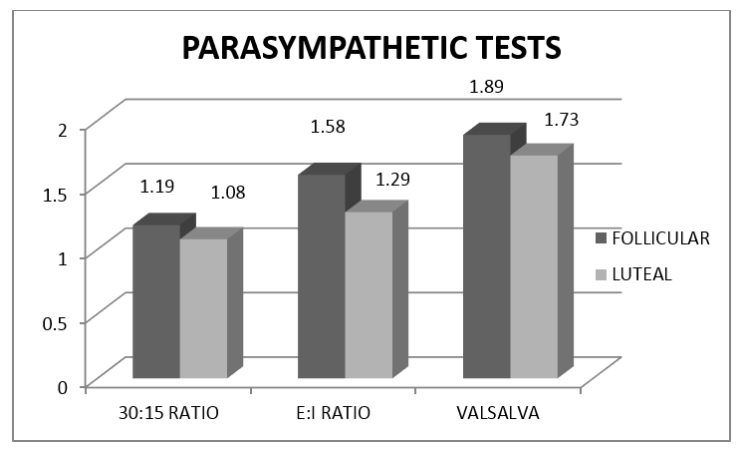

Graph 1 the above graph depicts the results of the parasympathetic subtests which clearly indicate that in all the subtests the values are statistically higher in the follicular phase, proving an increased vagal outflow during it.

\section{Discussion}

The current study was designed to assess the complex interactions of the gonadal steroids with the cardiovascular and hemodynamic regulatory systems. The results of the present study showed that the resting heart rate, SBP as well as DBP were higher during the luteal phase which points towards sympathetic dominance during the luteal phase. The battery of parasympathetic testse specially the 30:15 ratio, E:I ratio and Valsalva ratio conducted showed a blunted vagal outflow towards the luteal phase while the follicular phase shows parasympathetic predominance. The findings are concurrent with a study wherein they demonstrated a decrease in sympathetic neural trafficking along with an associated up regulation of vagal activity in response to hypertensive stimuli on injecting estrogen into Nucleus tractus solitarius which is a brainstem nuclei associated with the central autonomic cardiovascular control. ${ }^{14}$

Resting Heart rate and Blood pressure: It was observed that the baseline heart rate and blood pressure was higher in the luteal phase which is characterized by increase in the levels of progesterone which stimulates the HPA axis leading to activation of the sympathetic nervous activity. Progesterone alone can increase the 
cardiac excitability and its inherent thermogenic property leads to increase in the SA nodal discharge as a result of increased body core temperature. ${ }^{12,13}$ Progesterone is known to cause peripheral vasoconstriction by acting on the receptors present on the vascular smooth muscles leading to increased DBP. Increased translocation of blood in the form of increased venous return leads to increased SBP. Our results are similar to the study conductedwherein they used measurement of plasma concentration of norepinephrine which serves as a measure of sympathetic activity, the results pointed towards raised sympathetic activity during the luteal phase. ${ }^{14}$

Standing is to lying ratio (Postural Index): This test involvesstimulation carotid as a result of fall in blood pressure leading to reflex tachycardia and peripheral vasoconstriction. This immediate change observed in heart rate on standing is due to vagal withdrawal. Change in posture subjects an individual to orthostatic stress and checks for the integrity of the vasomotor system. Peripheral venous pooling which occurs as a result of standing causes inhibition of the carotid baroreceptors which now inhibits the cardiac vagal center and activates the vasomotor center which in turn causes transient tachycardia and vasoconstriction as well as venoconstriction. ${ }^{1}$ Initial rise in heart rate which is observed upon standing is due to parasympathetic withdrawal which reflects as transient tachycardia over the next 15 seconds which is then followed by rebound bradycardia which sets in at 30 seconds which occurs as a result of parasympathetic reactivation, this phenomenon of imposing orthostatic stress and the postural index checks for the intricacy and integrity of the vagal control on the cardiac autonomic structures. ${ }^{8}$

An elevated 30:15 ratio during the orthostatic stress test in the luteal phase signifies reduced parasympathetic activity during the same. The results of a study confirmed that estrogen when injected into the nucleus tractus solitarius which is a relay station for all cardiac autonomic afferents as well as efferentsshowed an increase in vagal tone and simultaneous reduction in sympathetic activity, hence estrogen secreted during the follicular phase has a role in up regulation of parasympathetic activity. ${ }^{15}$ Our study reports are coherent with the results of another study wherein they conducted the assessment of the sympathetic neural activity and observed a statistically higher sympathetic activity towards the luteal phase of menstrual cycle. ${ }^{16}$

Sinus arrhythmia: Both the cardiac as well as the respiratory system functions oscillate in unison both almost forming a single unit wherein there is increase in the heart rate during inspiration and heart rate decelerates during expiration, this phenomenon is known as sinus arrhythmia which occurs as a result of fluctuations in the vagal tone. This phenomenon occurs because the parasympathetic outflow to the heart fluctuates according to the phase of respiration. ${ }^{1,17}$ The stretch receptors in the lung are innervated by vagusand during inspiration the impulses from the same inhibit the cardio-inhibitory area in the medulla as a result of which there is acceleration of the heart rate during inspiration while the during expiration the stretch receptors are inhibited which results in activation of cardiac vagal center, apart from this respiratory impulses tend to centrally irradiate to the cardiovascular center located in the brain and brainstem and is also facilitated by respiratory reflexes which arise in the periphery. ${ }^{1,17}$ The phenomenon is most pronounced when the breathing is patterned at the rate of 6 breaths /min. ${ }^{18,19}$

The results of the current study show that the sinus arrhythmia represented by the E:I ratio was more pronounced during the follicular phase which confirms vagal dominance during it.

Valsalva Index: Valsalvamanoeuvre is a voluntary forced expiration of a subject against closed glottiswhich was described in 1794 by Antonio M.Valsalva, an Italian Anatomist for expelling pus from the middle ear by expiration against a closed glottis. ${ }^{20}$ An increase in intrathoracic pressure with increase in strain leads to transient increase in blood pressure (phase I), with no change in heart rate. In this phase of straining due to limited venous return, blood pressure decreases which is associated with parallel increase in heart rate which initially is due to vagal withdrawal followed by sympathetic activation.(phase II). With the release of strain (phase III), atransient fall in blood pressure due to mechanical displacement of blood into the pulmonary circulation which was initially under stress while heart rate does not change much.. In phase IV, due to further release in stress there occurs, an increase inblood pressure with concomitant bradycardia. Valsalva ratio is derived from the longest $\mathrm{R}-\mathrm{R}$ interval in phase IV divided by the shortest R-R interval in phase II.

Increased estrogen during the follicular phase is supposed to cause vagal activation along with a concomitant decline in the sympathetic activity. ${ }^{15,21} \mathrm{~A}$ study conducted proved an increased sympathetic activity in luteal phase with no changes in parasympathetic activity. ${ }^{22}$ There is evidence which proves that the Valsalva test elicits certain hemodynamic changes which lead to activation of both the sympathetic as well as parasympathetic nervous systems both at central and peripheral levels. ${ }^{(17)}$ Our results show concurrence with the results of studies wherein the resultant data proved that the sympathetic activity increased during the luteal phase..$^{22,23}$

\section{Conclusion}

The results of our study prove a definitealteration in the functioning of the autonomic nervous system with an increase in the sympathetic tone towards the luteal phase and reduced vagal activity. 


\section{Implications}

Parasympathetic attenuation or sympathetic hyperactivity in the form of autonomic dysfunction underlies the onset of most of the chronic cardiovascular diseases. The rising levels of estrogen which dominates the follicular phase causes vagal activation while progesterone which tends to dominate the luteal phase tends to antagonize the cardioprotective effects conferred upon by estrogen thus subjecting a female to higher propensity to develop cardiac abnormalities in the form of arrhythmias, myocardial ischemia, angina etc.Hence females stand a risk of development of cardiac abnormalities more so during the latter half of the menstrual cycle i.e.- luteal phase with increase in the percentage of morbidity and mortality. Thecomplexity of this interaction is exemplified from the results of the present study which will help in establishing a link in establishing treatment modalities or exercise regimens which can be cycle specific.

\section{Limitations \& Future Scope}

The actual hormone estimation was not conducted due to financial as well as technical constraints. The study can be extended to male counterparts as it will give an insight into the effect of testosterone on autonomic nervous system.

\section{Acknowledgement}

The author would like to thank Mr.Mallapur, statistician who helped analyze the data, the participants for their co-operation and the technical staff for their support.

\section{References}

1. Guyton and Hall. Female Physiology before Pregnancy and Female Hormones. In: 12th edition, Textbook of Medical Physiology. Philadelphia: Elsevier 2011:9871002.

2. Barrett K.E, Brooks H, Boitano S, Barman S.M. Reproductive Development and Function of the Female Reproductive System. In: 24th edition, Ganong's Review of Medical Physiology. New Delhi: McGraw Hill 2012:391-418.

3. Williams. Pregnancy planning and antepartum management. Text book of obstetrics. $22^{\text {nd }}$ edn. McGrawHill 2006:p. 226.

4. Perrot Applanat M. Estrogen receptors in the cardiovascular system. Steroids 1996:61:212-5

5. Mehta V: Autonomic function in different phases of menstrual cycle: Indian Jr. of Physiol. Pharmacol: 1993,37:56-8.

6. Gautam S, Shankar N, Tandon OP, Goel N. Comparison of cardiac autonomic functions among postmenopausal women with and without hormone replacement therapy, and premenopausal women. Indian J Physiol Pharmacol. 2011Oct-Dec;55(4):297-303.

7. Ewing DJ. Cardiovascular reflexes and autonomic neuropathy. Clin. Sci. Mol. Med. 1978;55:321-7.
8. Ewing DJ, Hume L, et al. Autonomic mechanism in the initial heart rate response to standing. J. Applied Physiol. 1980;49:809- 14.

9. Brost C, et al. Mechanism of initial heart rate response to postural change. Am. J. Physiol. 1982;243:H676-681.

10. Ewing, D.J. \& Clarke, B.F. Diagnosis and management of diabetic autonomic neuropathy. Br Med J 1982,285:9168.

11. Levin AB. A simple test of cardiac functions based up on the heart rate changes induced by Valsalva manoeuver. Am. J. Cardiol. 1966;18:90-9.

12. Lebrun CM, McKenzie DC, Prior JC, Taunton JE; Effects of menstrual cycle phase on athletic performance. Med Sci Sports Exerc, 1995;27(3):437-44.

13. Fletcher GF, Balady GJ, Amsterdam EA, Chaitman B, Eckel R, Fleg J, Froelicher VF, Leon AS, Pina IL, Rodney R, Simons-Morton DA, Williams MA, Bazzarre $\mathrm{T}$; Exercise standards for testing and training: a statement for healthcare professionals from the American Heart Association. Circulation, 2001; 104(14):1694-740.

14. Minson CT, Halliwill JR, Young TM, Joyner MJ. 29 Feb 2000. Influence of the menstrual cycle on Sympathetic activity, baroreflex Sensitivity, and vascular transduction in young women. 1: Circulation. 101(8):862-8

15. Bernardi F, Genazzani AR; The brain: target and source for sex steroid hormones. Women"s health and menopause. 1st ed. Netherlands: Parthenon publishing group, 1999;137-43.

16. Anjali Nadir Bhat: Autonomic function in different phases of menstrual cycle: Indian Jr. of Physiology and allied sciences: 2000,54(4):184-90.

17. O'Brien $\mathrm{AD}$, et al. Heart rate variability in healthy subjects Effects of age and derivation of normal range for test for autonomic functions. Br. Heart J. 1986;55:348-54

18. Piepoli M, Sleight P, Leuzzi S, Valle F, Spadacin G, Passino G, Johnston J, Bernardi L; Origin of Respiratory Sinus Arrhythmia in Conscious Humans. An Important Role for Arterial Carotid Baroreceptors. Circulation, 1997;95(7):1813-1.

19. Eckberg DL; The human respiratory rate. J Physiol,2003;54(2):339-52.

20. Bhagat AR, et al. The Valsalvamaneuver. J. of Asso. Physicians of India.1990;38:221-3.

21. Hirshoren, Nir; Tzoran, Inna; Makrienko, Igor; Edoute, Yehuda; Plawner, Mikahl M; Itskovitz-Eldor, Joseph; Jacob, Giris. April 2002. Menstrual Cycle effects on the Neuro humoral \& Autonomic Nervous Systems Regulating the Cardiovascular System. 87(4):1569-75.

22. Guasti L, Grimoldi P, Mainardi LT, Petrozzino MR, Piantanida E, Garganico D, Diolisi A, Zanotta D, Bertolini A, Ageno W, Grandi AM, Cerutti S, and Venco A. Autonomic function and baroreflex sensitivity during a normal ovulatory cycle in humans. ActaCardiol 54:209 13,1999 .

23. Fuenmayor AJ, Ramirez L, Fuenmayor AM. Left ventricular function and autonomic nervous system balance during two different stages of the menstrual cycle.Int J Cardiol 2000;72: 243-6. 